\title{
ISSN 1997-5902
}

\section{Production et commercialisation de la patate douce [lpomoea batatas (L.) Lam. dans les environs de Bongor.}

\author{
Alain Ignassou DJINET ${ }^{1 *}$, Somé KOUSSAO ${ }^{2}$, Benoudjita NGARYAM ${ }^{1}$ \\ ${ }^{1}$ Ecole Normale Supérieure de Bongor, Département des Sciences de la Vie et de la Terre. BP 15 Bongor, Tchad \\ 2 Institut National de l'Environnement et de la Recherche Agricole Ouagadougou /Burkina Faso . \\ *Auteur correspondant : E-mail : alain_djinet@yahoo.fr Tel : +23566438543
}

Original submitted in on 22nd December 2018. Published online at www.m.elewa.org/journals/ on 31st May 2019 https://dx.doi.org/10.4314/jab.v137i1.6

\section{RÉSUMÉ}

Objectif : l'objectif principal de ce travail est d'étudier les pratiques agricoles sur la production de la patate douce en milieu paysan traditionnel et de sa commercialisation.

Méthodologie et résultats : Pour réaliser cette étude des enquêtes ont été menées auprès des producteurs de 4 localités aux alentours de la ville de Bongor en 2015 et 2016. Le choix des localités était essentiellement basé sur la production importante, l'accessibilité des villages et la disponibilité des producteurs.

Du résultat, il ressort que les producteurs ne produisent pas assez des boutures, ils en procurent auprès des multiplicateurs spéciaux de la localité ou des localités voisines ou des villages du Cameroun voisin. Dans la pratique culturale les buttes sont plus utilisées avec un écartement de $50 \mathrm{~cm}$ entre elles et les fumures minérales sont également utilisées pour améliorer le rendement. Cependant le rendement obtenu varie entre 2,6 et 13 t/ha. Les produits récoltés sont vendus aux commerçants et aux consommateurs et le gain annuel des producteurs varie entre 100.000 et $300.000 \mathrm{~F}$ CFA.

Conclusion et application des résultats : cette présente étude a permis de connaitre les pratiques agricoles paysannes sur la production de la patate douce, le circuit de commercialisation de la patate douce et les difficultés liées à ces pratiques. De ce fait, les résultats obtenus permettront d'améliorer les pratiques de la culture de la patate douce adoptées par les producteurs ainsi augmenter le rendement et leurs revenus.

Mots clés : Patate douce, technique culturale, rendement, gain annuel

\begin{abstract}
Objective: The main objective of this work is to study agricultural practices on traditional sweet potato production and marketing.

Methodology and results: To carry out this study, surveys were conducted among producers in 4 localities around the city of Bongor in 2015 and 2016. The choice of localities was mainly based on the important production, the accessibility of the villages and the availability of the producers. As a result, it appears that producers do not produce enough cuttings; they get them from special multipliers of the locality or neighboring localities and villages of neighboring Cameroon. In the cultural practice the mounds are used with a distance of $50 \mathrm{~cm}$ between them and mineral fertilizers are also used to improve the yield. The yield
\end{abstract}


obtained varies between 2,6 and 13 tha. The harvested products are sold to consumers and traders and the annual gain of producers varies between 10.000 and 300.000 FCFA

Conclusion and application of results: this study has revealed peasant farming practices on sweet potato production, the sweet potato marketing system and the difficulties associated with this practices. As a result, the results obtained will improve the sweet potato crop practices adopted by the growers and increase the yield and their income.

Keywords: sweet potato, cultural practice, yield

\section{INTRODUCTION}

La patate douce Ipomoea batatas (L.)Lam est une convolvulacée cultivée dans les zones tropicales et subtropicales (Kaola et al., 2013). Elle est une culture simple, peu exigeante et arrive à survivre là où les autres cultures comme le maïs meurent; elle acquiert aussi moins de main d'œuvre que la plupart des autres cultures vivrières. Les caractéristiques agronomiques telles qu'une large adaptabilité, une forte productivité, un cycle court et une forte valeur nutritionnelle font de la patate douce une culture particulièrement importante pour la sécurité alimentaire des pays soumis à de fortes pressions anthropiques et vulnérable au changement climatique (Bovell-Benjamin, 2007). Sa culture peut être échelonnée toute l'année avec des prélèvements des boutures d'un champ à l'autre, mais elle est aussi soumise à beaucoup de contraintes. De nos jours, la patate douce bénéficie de programme d'amélioration (Vernier et Varin, 1994). Selon la division de la statistique agricole du ministère de l'agriculture en 2009, au Tchad la production est passée de 57658 tonnes durant la campagne 2006/2007 à 43342 tonnes au cours de la campagne 2007/2008. Cette baisse de production montre que cette culture n'est pas assez pratiquée. Depuis quelques années les populations rurales et périurbaines de Bongor commencent à s'intéresser à la culture de la patate douce qui autre fois était considérée comme une culture de case, elle est réservée aux femmes et bien indiquée pour traverser la période de soudure. Aujourd'hui, elle est devenue une source de revenu pour la population rurale tant urbaine. A la récolte les produits sont évacués vers les grands centres où les besoins sont énormes. L'objectif de ce présent travail est d'étudier les pratiques agricoles sur la production de la patate douce en milieu paysan traditionnel et de sa commercialisation afin de cerner avec exactitude les problèmes qui en découlent et de mieux connaitre le circuit de commercialisation de la patate douce pour lui donner sa place dans l'échiquier agricole.

\section{MATERIALS AND METHODS}

Matériel végétal : L'étude a porté sur les variétés locales de patate douce cultivées dans les villages aux alentours de Bongor.
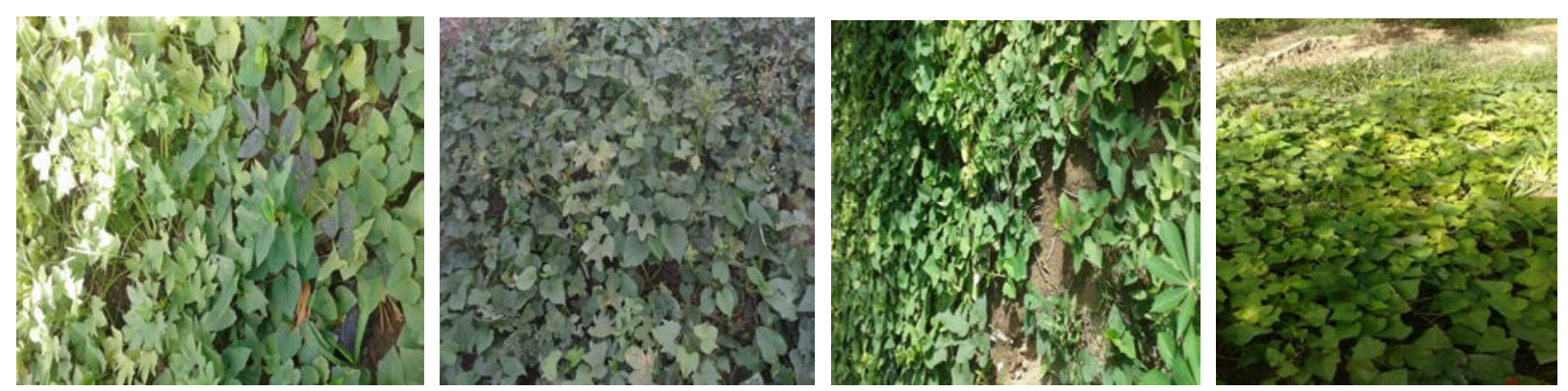

Photo 1 : matériel végétal en pré pépinière dans le jardin des producteurs 
Méthode : L'étude a utilisé une enquête faite auprès des producteurs de la patate douce dans les environs de Bongor. Pour mener cette étude quatre(4) villages ont été retenus. Le choix des villages a été réalisé avec le concours des étudiants de la troisième année des Sciences de la Vie et de la Terre. Les critères du choix étaient essentiellement : la production importante de la patate douce, accessibilité du village et la disponibilité des producteurs. Ainsi quatre(4) villages ont été retenus et par village un échantillon de 15 producteurs a été enquêté. Les enquêtes ont été menées en 2015 et 2016.

Traitement des données: Les données ont été soumises à une analyse de variance(ANOVA) à l'aide $\mathrm{du}$ logiciel XLSTAT version 7.5.2. Les tests de comparaison des moyennes ont été effectués selon la méthode de Fisher au seuil de $5 \%$.
Site d'étude : La région du Mayo-Kebbi Est l'une des 23 régions du Tchad dont le Chef-lieu est Bongor. La ville de Bongor est située à $10^{\circ} .28$ latitude Nord et à $15^{\circ} 37$ longitude Est. Le climat est de type soudanien caractérisé par une saison sèche très longue et une saison des pluies qui dure environ 4 mois avec une hauteur moyenne des pluies qui oscille entre $600 \mathrm{~mm}$ et $900 \mathrm{~mm}$ par an.

Le sud, l'Ouest et le Nord-Ouest de la ville sont des zones inondées par contre l'Est et le nord Est sont des zones exondées. Dans les zones inondées, la culture du riz y est présente tandis que dans les zones exondées, la culture de maïs, arachides, sésames, mil et autres est importante. C'est aussi dans ces zones exondées que la culture de la patate douce est très développée. Les localités qui ont fait l'objet de cette enquête sont situées au nord et à l'Est de la ville de Bongor.

Tableau 1 : les localités productrices de la patate douce au tour de la ville de Bongor

\begin{tabular}{|l|l|}
\hline Localité & \multicolumn{1}{c|}{ Localisation } \\
\hline Bariam & Au nord de Bongor à $15 \mathrm{Km}$ \\
\hline Gournaïda & Au nord de Bongor à $25 \mathrm{Km}$ \\
\hline Guizedé & A l'Est de Bongor à $12 \mathrm{Km}$ \\
\hline Moulkou & Au nord de Bongor à $40 \mathrm{Km}$ \\
\hline
\end{tabular}

\section{RESULTATS}

Production de matériel végétal : Le matériel végétal utilisé dans la production de la patate douce est appelé boutures ou semence ou lianes selon les milieux. Le tableau 2 présente la production du matériel végétal dans les différentes localités. Le matériel végétal utilisé par les producteurs provient soit du Cameroun, soit de la localité soit des localités voisines. $64,15 \%$ des producteurs enquêtés déclarent que les boutures utilisées proviennent du Cameroun. Les producteurs de Bariam utilisent plus des boutures venant du Cameroun soit $79,99 \%$. Par contre $25,82 \%$ des enquêtés disent que les boutures sont produites dans les localités. Les producteurs de Gournaida soit $46,66 \%$ affirment qu'ils produisent aussi eux-mêmes des boutures. Cependant $10,82 \%$ des producteurs enquêtés disent également que les boutures viennent du Cameroun voisin et sont produits aussi localement. Les producteurs de Guizedé sont réputés pour cette pratique. S'agissant du nombre de variétés cultivées, $29,15 \%$ des producteurs utilisent plus de 3 variétés. C'est à Moulkou que les producteurs cultivent un nombre important des variétés soit 49,99 $\%$. En outre, $82,49 \%$ des producteurs disent que les boutures sont obtenues chez certains producteurs et les jardiniers spécialisés dans le domaine. Par ailleurs, les boutures produites par les producteurs ou les multiplicateurs de la place sont conservées soit à partir des tubercules enfouis dans le sol $(52,49 \%)$ soit à partir des tiges mises en pépinières $(47,49 \%)$. Les producteurs $(66,66 \%)$ de Bariam préfèrent multiplier les boutures à partir des tubercules gardés dans le sol. Par contre $73,33 \%$ des producteurs de Gournaida ont une préférence pour la tige pour pouvoir multiplier les boutures. Les pépinières sont conservées dans les enclos aménagés derrière les cases $(93,82 \%)$ contre $6,66 \%$ dans les champs. A Moulkou tous les producteurs $(100 \%)$ apprécient la technique de conservation des boutures dans les enclos. Tandis qu'à Bariam elles sont conservées au champ. II faut noter que les boutures sont conservées pendant 1 à 3 mois avant d'être récoltées et plantées. 39,99 \% des producteurs disent que la pépinière est entretenue pendant 3 mois. La localité la plus indiquée pour cette conservation est Bariam soit 59,99 \%. Et 44,99 \% des producteurs affirment que la récolte se fait au mois de juillet période de plantation. C'est pendant cette période que la plupart des producteurs de Bariam récoltent les boutures en vue de la plantation 
Tableau 2 : production du matériel végétal

\begin{tabular}{|c|c|c|c|c|c|c|c|c|}
\hline $\begin{array}{l}\text { matériel } \\
\text { végétal }\end{array}$ & & F Fisher & $\begin{array}{l}\mathrm{P} \\
\text { associées }\end{array}$ & Bariam & Gournaida & Guizede & Moulkou & Moy \\
\hline \multirow[b]{3}{*}{ PB } & Cameroun & 4,38 & 0,094 & $79,99^{a}$ & $49,99^{b}$ & $66,66^{\mathrm{ab}}$ & $59,99 \mathrm{ab}$ & 64,15 \\
\hline & Locale & 31,40 & 0,003 & $9,99^{c}$ & $46,66^{a}$ & $16,66^{c}$ & $29,99^{b}$ & 25,82 \\
\hline & $\begin{array}{l}\text { Locale et } \\
\text { Cameroun }\end{array}$ & 2,11 & 0,24 & $9,99^{a}$ & $6,66^{a}$ & $16,66^{a}$ & $9,99 a$ & 10,82 \\
\hline \multirow{3}{*}{ NV } & $>3$ & 11,56 & 0,019 & $19,99^{b}$ & $29,99 \mathrm{~b}$ & $16,66^{b}$ & $49,99 a$ & 29,15 \\
\hline & 3 & 3,57 & 0,12 & $23,33^{b}$ & $36,66^{\mathrm{ab}}$ & $33,33^{\mathrm{ab}}$ & $43,33^{a}$ & 34,16 \\
\hline & 2 & 29,75 & 0,003 & $56,66^{a}$ & $33,33^{b}$ & $49,99 a$ & $6,66^{c}$ & 36,66 \\
\hline \multirow[b]{2}{*}{ MOB } & Producteur & 1,1 & 0,44 & $26,66^{a}$ & $13,33^{a}$ & $16,66^{a}$ & $13,33^{a}$ & 17,49 \\
\hline & Jardiniers & 1,1 & 0,44 & $73,33^{a}$ & $86,66^{a}$ & $83,33^{a}$ & $86,66^{a}$ & 82,49 \\
\hline \multirow[t]{2}{*}{ MCB } & $\begin{array}{l}\text { Tubercules } \\
\text { gardés }\end{array}$ & 8,69 & 0,032 & $66,66^{a}$ & $26,66^{b}$ & $56,66^{a}$ & $59,99 a$ & 52,49 \\
\hline & Pépinières & 8,69 & 0,032 & $33,33^{b}$ & $73,33^{a}$ & $43,33^{b}$ & $39,99^{b}$ & 47,49 \\
\hline \multirow[b]{2}{*}{ LCB } & enclos & 2,22 & 0,22 & $86,66^{a}$ & $96,66^{a}$ & $89,99^{a}$ & $100^{a}$ & 93,32 \\
\hline & champ & 2,22 & 0,22 & $13,33^{a}$ & $3,33^{a}$ & $9,99^{a}$ & $00^{\mathrm{a}}$ & 6,66 \\
\hline \multirow{3}{*}{ DCB } & 1 mois & 1,22 & 0,41 & $16,66^{a}$ & $30^{\mathrm{a}}$ & $23,33^{a}$ & $16,66^{a}$ & 21,66 \\
\hline & 2 mois & 15,08 & 0,012 & $23,33^{b}$ & $66,66^{a}$ & $23,33^{b}$ & $39,99^{b}$ & 38,32 \\
\hline & 3 mois & 12,88 & 0,016 & $59,99^{a}$ & $3,33^{b}$ & $53,33^{a}$ & $43,33^{a}$ & 39,99 \\
\hline \multirow{4}{*}{ PRB } & Mai & 23,34 & 0,005 & $3,33^{b}$ & $29,99^{a}$ & $00^{b}$ & $39,99^{a}$ & 18,32 \\
\hline & Juin & 3,33 & 0,13 & $16,66^{\mathrm{ab}}$ & $13,33^{\mathrm{ab}}$ & $9,99 \mathrm{~b}$ & $20^{a}$ & 14,99 \\
\hline & Juillet & 2,11 & 0,24 & $56,66^{a}$ & $43,33^{a}$ & $43,33^{a}$ & $36,66^{a}$ & 44,99 \\
\hline & Aout & 5,81 & 0,061 & $23,33^{a b}$ & $13,33^{b}$ & $46,66^{a}$ & $16,66^{b}$ & 24,99 \\
\hline
\end{tabular}

Les chiffres ayant la même lettre en exposant ne sont pas significativement différents.

PB : Provenance des boutures ; NV : Nombre des variétés ; MOB : Mode d'obtention des boutures MCB : Mode de conservation des boutures; LCB : Lieu de conservation des boutures ; DCB : Durée de conservation des boutures ; PRB : Période de récolte des boutures.

Technique culturale (Culture de la patate douce): Le tableau 3 présente la technique de cultures de la patate douce adoptées par les producteurs. Ils utilisent soit des billons, soit des buttes, soit les deux. Les écartements entre les billons ou les buttes varient de 20 $\mathrm{cm}$ à $1 \mathrm{~m}$ selon les localités, le cycle de culture varie de 2 à 4 mois et le temps mis dans le domaine par les producteurs va de 5 ans à plus de 10 ans. S'agissant de la technique de bouturage, $62,65 \%$ des producteurs utilisent des buttes. C'est à Bariam et Gournaida que cette technique est beaucoup plus sollicitée par les producteurs soit 79,99\%. Cependant $19,99 \%$ préfèrent les billons. Comparativement aux producteurs des autres localités ceux de Moulkou préfèrent cultiver la patate douce sur des billons soit $26,66 \%$ des enquêtés. Malgré cette préférence pour les billons, ces derniers utilisent aussi des buttes soit $46,66 \%$ des enquêtés. S'agissant des écartements entre les billons ou buttes, la plupart des producteurs soit $57,49 \%$ ont une préférence pour un écartement de $50 \mathrm{~cm}$. C'est à Moulkou que les producteurs utilisent cet écartement soit $73,33 \%$ des enquêtés. Rares les écartements d' $1 \mathrm{~m}$ et $30 \mathrm{~cm}$ sont pris en compte dans la pratique paysanne. Pour le cycle de culture, il varie de 2 à 4 mois. 54,16\% des producteurs déclarent cultiver des variétés ayant le cycle de 3 mois. Comparativement aux producteurs des autres localités, ceux de Guizédé cultivent assez des variétés. Pour le temps mis dans l'activité, les producteurs ont une bonne expérience car ils ont 5 à plus de 10 ans dans le domaine. $62,49 \%$ des enquêtés disent qu'ils cultivent la patate douce depuis plus de 10 ans contre 13,32 \% qui sont dans le domaine depuis 5 ans. Les plus vieux producteurs de patate douce sont à Bariam soit 89,99 $\%$ des enquêtés. 
Tableau 3 : technique culturale

\begin{tabular}{|c|c|c|c|c|c|c|c|c|}
\hline & & F Fisher & $\mathrm{P}$ associées & Bariam & Gournaida & Guizede & Moulkou & Moy \\
\hline \multirow[t]{3}{*}{ TB } & $\begin{array}{l}\text { Billons et } \\
\text { buttes }\end{array}$ & 23,34 & 0,005 & $6,66^{b}$ & $3,33^{b}$ & $16,66^{b}$ & $46,66^{a}$ & 18,32 \\
\hline & billons & 0,74 & 0,58 & $13,33^{a}$ & $16,66^{a}$ & $23,33^{a}$ & $26,66^{a}$ & 19,99 \\
\hline & buttes & 8,14 & 0,035 & $79,99 a$ & $79,99 a$ & $59,99 \mathrm{ab}$ & $26,66^{b}$ & 62,65 \\
\hline \multirow{3}{*}{ EBB } & $1 \mathrm{~m}$ & 6,59 & 0,050 & $00^{b}$ & $9,99 \mathrm{ab}$ & $00^{b}$ & $19,99^{a}$ & 7,49 \\
\hline & 50 & 7,75 & 0,038 & $43,33^{a}$ & $56,66^{a b}$ & $56,66^{\mathrm{ab}}$ & $73,33^{a}$ & 57,49 \\
\hline & $30 \mathrm{~cm}$ & 50,16 & 0,001 & $43,33^{a}$ & $3,33^{b}$ & $36,66^{a}$ & $6,66^{b}$ & 22,49 \\
\hline \multirow{4}{*}{$\mathrm{CC}$} & 4 mois & 5,67 & 0,063 & $16,66^{a}$ & $13,33^{a b}$ & $3,33^{b}$ & $3,33^{b}$ & 9,16 \\
\hline & 3-4 mois & 61,36 & 0,001 & $36,66^{b}$ & $3,33^{d}$ & $16,66^{c}$ & $63,33^{a}$ & 29,99 \\
\hline & 3 mois & 7,41 & 0,041 & $39,99 \mathrm{bc}$ & $66,66^{a b}$ & $76,66^{a}$ & $33,3^{c}$ & 54,16 \\
\hline & 2 mois & 6,56 & 0,05 & $3,33^{b}$ & $16,66^{a}$ & $3,33^{b}$ & $00^{b}$ & 5,83 \\
\hline \multirow{3}{*}{ TMD } & $>10$ ans & 12,58 & 0,017 & $89,99 a$ & $53,33^{b c}$ & $66,66^{\mathrm{ab}}$ & $39,99 c$ & 62,49 \\
\hline & 10 ans & 6,33 & 0,053 & $9,99 \mathrm{~b}$ & $29,99^{a}$ & $23,33^{a}$ & $23,33^{a}$ & 21,66 \\
\hline & 5 ans & 22,63 & 0,006 & $00^{c}$ & $16,66^{b}$ & $9,99^{b}$ & $26,66^{a}$ & 13,32 \\
\hline
\end{tabular}

Les chiffres ayant la même lettre en exposant ne sont pas significativement différents.

TB : Technique de bouturage ; EBB : Ecartement entre les billons ou buttes ; CC : Cycle de culture ; TMD : Temps mis dans le domaine.

Utilisation des fumures dans la production de la patate douce: Le tableau 4 présente le type, la quantité de fumures utilisées par les producteurs dans la production de la patate douce afin d'améliorer leur rendement. Du résultat, il ressort que la majorité des producteurs $(85,52 \%)$ utilisent de fumure de types variables. A Moulkou les fumures sont beaucoup plus utilisées par les producteurs soit 96,66 \%. Cependant la quantité de fumures utilisées par les producteurs varie d'un milieu à un autre. $36,35 \%$ des producteurs utilisent $20 \mathrm{~kg}$ de fumures à l'hectare contre $22,98 \%, 14,31 \%$ et $20,94 \%$ respectivement pour $50 \mathrm{~kg}, 30 \mathrm{~kg}$ et $10 \mathrm{~kg}$ à l'hectare. Les producteurs de Gournaida manifestent une préférence pour $20 \mathrm{~kg}$ de fumures à l'hectare soit $67,82 \%$. En outre, les fumures utilisées sont soit de nature organique, soit de nature minérale. Certains producteurs utilisent les deux à la fois. Mais la majorité des producteurs enquêtés $(81,13 \%)$ utilisent la fumure minérale pour améliorer leurs rendements contre $5,39 \%$ qui ont une préférence pour la fumure organique et $14,04 \%$ qui ont aussi une préférence pour les deux. C'est à Gournaida que la fumure minérale est souvent utilisée par les producteurs dans la production de la patate douce.

Tableau 4 : utilisation de fumures dans la production de la patate douce

\begin{tabular}{|l|l|c|c|c|c|c|c|c|}
\hline & & F Fisher & P associées & Bariam & Gournaida & Guizede & Moulkou & \\
\hline \multirow{4}{*}{ UF } & Oui & 1,71 & 0,30 & $79,99^{\mathrm{a}}$ & $79,99^{\mathrm{a}}$ & $86,66^{\mathrm{a}}$ & $96,66^{\mathrm{a}}$ & 85,82 \\
\cline { 2 - 9 } & non & 1,71 & 0,30 & $19,99^{\mathrm{a}}$ & $19,99^{\mathrm{a}}$ & $13,33^{\mathrm{a}}$ & $3,33^{\mathrm{a}}$ & 14,16 \\
\hline \multirow{4}{*}{ QFU } & 11,21 & 0,22 & $13,33^{\mathrm{bc}}$ & $3,84^{\mathrm{c}}$ & $26,66^{\mathrm{ab}}$ & $48,09^{\mathrm{a}}$ & 22,98 \\
\cline { 2 - 9 } & $50 \mathrm{~kg}$ & 7,77 & 0,038 & $6,66^{\mathrm{b}}$ & $16,78^{\mathrm{ab}}$ & $23,33^{\mathrm{a}}$ & $10,47^{\mathrm{b}}$ & 14,31 \\
\cline { 2 - 9 } & $20 \mathrm{~kg}$ & 6,80 & 0,047 & $43,33^{\mathrm{ab}}$ & $67,82^{\mathrm{a}}$ & $13,33^{\mathrm{b}}$ & $20,95^{\mathrm{b}}$ & 36,35 \\
\cline { 2 - 9 } & $10 \mathrm{~kg}$ & 4,27 & 0,09 & $33,33^{\mathrm{a}}$ & $00^{\mathrm{a}}$ & $36,66^{\mathrm{a}}$ & $13,80^{\mathrm{ab}}$ & 20,94 \\
\hline \multirow{4}{*}{ TF } & Organique & 0,79 & 0,55 & $3,33^{\mathrm{a}}$ & $3,84^{\mathrm{a}}$ & $4,16^{\mathrm{a}}$ & $10,23^{\mathrm{a}}$ & 5,39 \\
\cline { 2 - 9 } & Minérale & 22,59 & 0,006 & $89,99^{\mathrm{a}}$ & $95,15^{\mathrm{a}}$ & $87,49^{\mathrm{a}}$ & $51,90^{\mathrm{b}}$ & 81,13 \\
\cline { 2 - 9 } & $\begin{array}{l}\text { Organique et } \\
\text { minérale }\end{array}$ & 68,96 & 0,001 & $9,99^{\mathrm{b}}$ & $00^{\mathrm{c}}$ & $8,33^{\mathrm{b}}$ & $37,85^{\mathrm{a}}$ & 14,04 \\
\hline
\end{tabular}

Les chiffres ayant la même lettre en exposant ne sont pas significativement différents.

UF : Utilisation de fumures; QFU : Quantité de fumures utilisées ; TF : Type de fumures. 
Récolte, rendement et conservation de la patate douce : La fréquence de récolte, le rendement et la conservation de la patate douce sont présentés dans le tableau 5. De ce tableau, on constate que la fréquence de récolte va de 2 jours à plus 10 jours. $29,15 \%$ des producteurs déclarent que la récolte se fait pendant 2 jours. Par contre $39,99 \%$ disent qu'elle se fait à plus de 10 jours. Comparativement aux autres localités c'est à Moulkou que la fréquence de récolte atteint 10 jours. $63,33 \%$ des producteurs en témoignent. S'agissant du nombre d'hectare cultivé par les producteurs, il varie entre $1 / 4$ et 2 hectares. $49,99 \%$ des producteurs affirment qu'ils cultivent la patate douce dans une superficie d'environ 2 hectares contre 9,99\% qui utilisent $1 / 4$ d'hectare comme superficie pour la production de la patate douce. La localité dont les producteurs occupent une superficie importante est Moulkou soit 73,33\%. Cependant le nombre de sac obtenus par les producteurs à la récolte varie entre 20 et 100 sacs à l'hectare soit 2,6 et 13 tonnes à I'hectare. Le rendement à Bariam semble être important par rapport aux autres localités. En outre, $72,49 \%$ des producteurs ne conservent pas les tubercules récoltés contre $27,49 \%$ qui les conservent. Les producteurs qui conservent le font au champ ou dans un autre endroit. $34,57 \%$ affirment qu'ils conservent les tubercules au champ. Les tubercules non conservés sont directement vendus et consommés. 16,66 \% des producteurs vendent les tubercules après la récolte et $8,32 \%$ les consomment. Par contre $76,66 \%$ des producteurs affirment qu'ils vendent et consomment. A guizédé $56,66 \%$ des producteurs vendent aussitôt leurs produits après la récolte et $16,66 \%$ les consomment également. Par ailleurs, la patate récoltée est consommée sous forme frite, bouillie. Dans certaines localités les deux formes sont appréciées. 59,16\% des producteurs déclarent que la patate est consommée sous les deux formes contre $29,16 \%$ sous forme bouillie et $11,66 \%$ sous forme frite. A Guizedé, elle est consommée sous forme bouillie $(53,33 \%)$. Tandis qu'à Gournaida et Moulkou, la patate est consommée sous les deux formes (frite et bouillie) soit 93, 33\%.

Tableau 5 : récolte, rendement et conservation de la patate douce

\begin{tabular}{|c|c|c|c|c|c|c|c|c|}
\hline & & $\begin{array}{l}\text { F } \\
\text { Fisher }\end{array}$ & $\begin{array}{l}\mathrm{P} \\
\text { associée } \\
\mathrm{s}\end{array}$ & Bariam & Gournaida & Guizede & Moulkou & \\
\hline \multirow[b]{3}{*}{ FR } & 10 jours & 13,07 & 0,016 & $39,99^{b}$ & $39,99^{b}$ & $16,66^{c}$ & $63,33^{a}$ & 39,99 \\
\hline & 7 jours & 7,76 & 0,038 & $29,99 \mathrm{ab}$ & $29,99 \mathrm{ab}$ & $46,66^{a}$ & $16,66^{b}$ & 30,82 \\
\hline & 2 à 3 jours & 1,13 & 0,43 & $29,99^{a}$ & $29,99 a$ & $36,66^{a}$ & $19,99 a$ & 29,15 \\
\hline \multirow{4}{*}{$\mathrm{NHCP}$} & 2 ha & 20,51 & 0,007 & $56,66^{a}$ & $53,33^{a}$ & $16,66^{b}$ & $73,33^{a}$ & 49,99 \\
\hline & $1 \mathrm{ha}$ & 3,86 & 0,11 & $23,33^{a b}$ & $19,99^{a b}$ & $39,99 a$ & $16,66^{b}$ & 24,99 \\
\hline & $1 / 2 \mathrm{ha}$ & 19,66 & 0,07 & $3,33^{b}$ & $9,99^{b}$ & $36,66^{a}$ & $9,99^{b}$ & 14,99 \\
\hline & $1 / 4 \mathrm{ha}$ & 3,99 & 0,107 & $16,66^{a}$ & $16,66^{a}$ & $6,66^{\mathrm{ab}}$ & $00^{b}$ & 9,99 \\
\hline \multirow{3}{*}{ NSOPH } & $40-50$ & 5,11 & 0,07 & $66,66^{a}$ & $46,66^{\mathrm{ab}}$ & $29,99^{b}$ & $36,66^{b}$ & 44,99 \\
\hline & $30-40$ & 6,62 & 0,050 & $23,33^{b}$ & $36,66^{a b}$ & $46,66^{a}$ & $23,33^{b}$ & 32,49 \\
\hline & $20-30$ & 3,66 & 0,12 & $3,33^{b}$ & $9,99^{b}$ & $36,66^{a}$ & $9,99^{b}$ & 14,99 \\
\hline \multirow[b]{2}{*}{ CTR } & Oui & 18,58 & 0,008 & $13,33^{b}$ & $6,66^{b}$ & $13,33^{b}$ & $76,66^{a}$ & 27,49 \\
\hline & non & 18,58 & 0,008 & $86,66^{a}$ & $93,33^{a}$ & $86,66^{a}$ & $23,33^{b}$ & 72,49 \\
\hline \multirow[t]{2}{*}{ LC } & Champ & 75,48 & 0001 & $41,66^{b}$ & $00^{c}$ & $9,99^{c}$ & $86,66^{a}$ & 34,57 \\
\hline & autres & 24,95 & 0,005 & $20,83^{a}$ & $00^{b}$ & $00^{b}$ & $00^{\mathrm{b}}$ & 5,20 \\
\hline \multirow[b]{3}{*}{ DTAR } & vente & 131,86 & 0,000 & $9,99^{b}$ & $00^{c}$ & $56,66^{a}$ & $00^{c}$ & 16,66 \\
\hline & consommation & 2,88 & 0,16 & $9,99 \mathrm{ab}$ & $6,66^{\mathrm{ab}}$ & $16,66^{a}$ & $00^{b}$ & 8,32 \\
\hline & $\begin{array}{l}\text { Vente et } \\
\text { consommation }\end{array}$ & 108,07 & 0,000 & $79,99 b$ & $100^{a}$ & $26,66^{c}$ & $100^{a}$ & 76,66 \\
\hline \multirow{3}{*}{ FCT } & frite & 37,95 & 0002 & $29,99^{a}$ & $00^{c}$ & $16,66^{b}$ & $00^{\circ}$ & 11,66 \\
\hline & bouillie & 18,74 & 0,008 & $49,99 a$ & $6,66^{\mathrm{b}}$ & $53,33^{a}$ & $6,66^{\mathrm{b}}$ & 29,16 \\
\hline & Frite et bouillie & 43,54 & 0,002 & $19,99^{b}$ & $93,33^{a}$ & $29,99^{b}$ & $93,33^{a}$ & 59,16 \\
\hline
\end{tabular}

Les chiffres ayant la même lettre en exposant ne sont pas significativement différents. 
FR : Fréquence de récolte ; NHCP : Nombre d'hectare cultivé par le producteur ; NSOPH : Nombre de sacs obtenus par le producteur à l'hectare ; CTR : Conservation des tubercules récoltés ; LC : Lieu de conservation ; DTAR :

Destination des tubercules après récolte ; FCT : Forme sous laquelle est consommé le tubercule.

Appréciation des acheteurs et consommateurs : Les appréciations des acheteurs et consommateurs de la patate douce dans les différentes localités faisant l'objet d'étude sont présentées sur la figure 1. Les éléments d'appréciation sont bons tubercules (forme); couleur de l'enveloppe et le cycle court; de bons tubercules et cycle court; la teneur en sucre importante ; bons tubercules, cycle court et teneur en sucre importante; bons tubercules et couleur de l'enveloppe; bons tubercules et matière sèche importante ; le cycle court ; bons tubercules, couleur de l'enveloppe et bouture facile à trouver. Du résultat, il ressort que les appréciations varient d'une localité à une autre. Les acheteurs et les consommateurs de Gournaida préfèrent des bons tubercules $(53,33 \%)$. Par contre ceux de Bariam et Guizédé ont une préférence pour des bons tubercules et s'intéressent aussi à la couleur de l'enveloppe soit respectivement $59,99 \%$ et $29,99 \%$. Tandis qu'à Moulkou, ces derniers s'intéressent aux bons tubercules ayant une matière sèche importante $(29,99 \%)$.

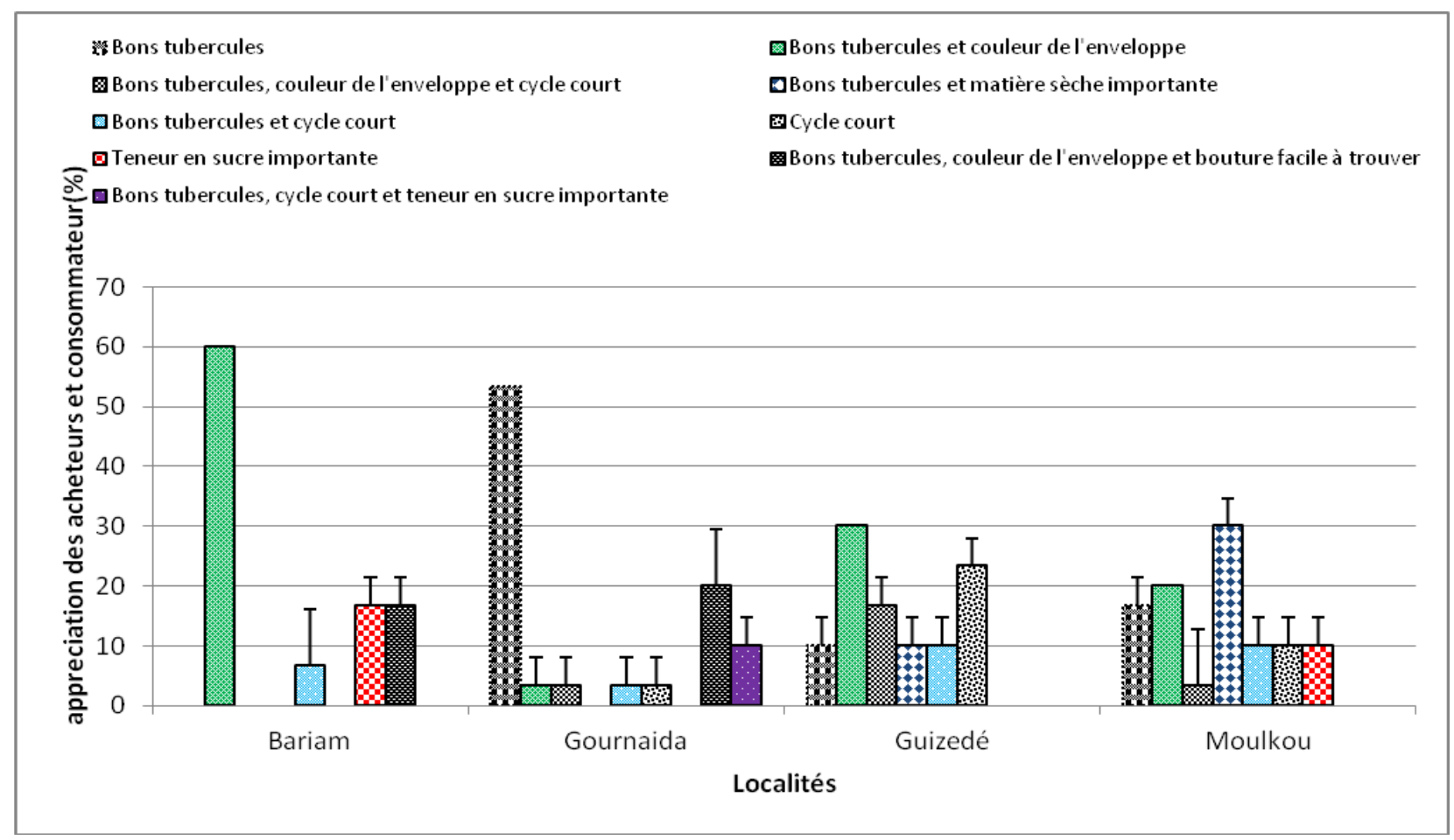

Figure 1 : appréciation des acheteurs et consommateurs de patate douce

Commercialisation de la patate douce : Le tableau 6 présente la vente et le gain obtenu par le producteur après la récolte. Du tableau, il ressort que, la vente se fait soit par le producteur lui-même, soit par le producteur et sa femme soit par sa femme seule. 54,99 $\%$ des producteurs vendent eux même le produit de récolte. Cependant $35,82 \%$ déclarent qu'ils font la vente avec leurs femmes. Par contre $8,32 \%$ des producteurs confient cette tache uniquement à leurs femmes. Par ailleurs, $58,32 \%$ des producteurs vendent leurs produits aux commerçants revendeurs venant de la ville. Tandis que $41,65 \%$ en dehors des commerçants revendeur vendent aussi leurs produits aux consommateurs. C'est à Bariam que la vente se fait plus aux commerçants revendeurs soit $73,33 \%$ des producteurs. En outre, les ventes se font soit sur les marchés de leur localité, soit sur les marchés de la ville. S'agissant de la vente, elle se fait soit par sacs, soit au volume. $60,82 \%$ des producteurs préfèrent la vente au volume contre $34,16 \%$ qui ont une préférence pour la vente par sac. À Gournaida tout comme à Moulkou, 93,33 \% des producteurs préfèrent vendre leurs produits au volume. Par contre ceux de Bariam aiment faire la vente par sac. Le prix du sac varie entre 
2000 et $10000 \mathrm{f}$. De toutes les localités, $78,33 \%$ des producteurs affirment que le prix du sac varie entre 5000 et $10000 \mathrm{~F}$ contre $21,66 \%$ qui disent que le prix varie entre 2000 et $5000 \mathrm{~F}$. Pour le prix au volume $33,32 \%$ des producteurs disent qu'il varie entre 200 et 500 contre $66,66 \%$ qui disent que le prix varie entre 50 et $200 \mathrm{~F}$. Le gain annuel des producteurs varie entre 100.000 et 300.000 f. $48,32 \%$ des producteurs affirment qu'ils gagnent plus de $300.000 \mathrm{f}$ pendant la campagne contre $5,82 \%$ qui gagnent moins de $100.000 \mathrm{f}$. C'est à Moulkou que les producteurs ont un gain annuel important.

Tableau 6 : commercialisation de la patate douce

\begin{tabular}{|c|c|c|c|c|c|c|c|c|}
\hline & 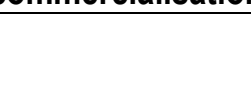 & $\begin{array}{l}\text { F } \\
\text { Fisher }\end{array}$ & $\begin{array}{l}\mathrm{P} \\
\text { associées }\end{array}$ & Bariam & Gournaida & Guizede & Moulkou & Moy \\
\hline \multirow{3}{*}{ VPD } & $\begin{array}{l}\text { Producteur lui } \\
\text { même }\end{array}$ & 6,53 & 0,051 & $56,66^{\mathrm{ab}}$ & $43,33^{b}$ & $73,33^{a}$ & $46,66^{b}$ & 54,99 \\
\hline & $\begin{array}{l}\text { Producteur et sa } \\
\text { femme }\end{array}$ & 12,33 & 0,017 & $29,99 \mathrm{bc}$ & $46,66^{\mathrm{ab}}$ & $16,66^{c}$ & $49,99 a$ & 35,82 \\
\hline & Sa femme & 0,99 & 0,47 & $9,99^{a}$ & $9,99^{a}$ & $9,99^{a}$ & $3,33^{a}$ & 8,32 \\
\hline \multirow[b]{2}{*}{ APD } & commerçants & 42,78 & 0,002 & $73,33^{a b}$ & $59,99^{b}$ & $89,99 a$ & $9,99^{c}$ & 58,32 \\
\hline & $\begin{array}{l}\text { Commerçants et } \\
\text { autres }\end{array}$ & 42,80 & 0,002 & $26,66^{b c}$ & $39,99^{b}$ & $9,99^{c}$ & $89,99^{a}$ & 41,65 \\
\hline \multirow{3}{*}{ LV } & $\begin{array}{l}\text { Marchés } \\
\text { hebdomadaires }\end{array}$ & 3,46 & 0,13 & $49,99^{a}$ & $46,66^{a}$ & $63,33^{a}$ & $66,66^{a}$ & 56,66 \\
\hline & $\begin{array}{l}\text { Marchés } \\
\text { hebdomadaire et } \\
\text { ville }\end{array}$ & 9,92 & 0,025 & $19,99^{b}$ & $53,33^{a}$ & $19,99^{b}$ & $9,99 \mathrm{~b}$ & 25,82 \\
\hline & $\begin{array}{l}\text { Marchés de la } \\
\text { ville }\end{array}$ & 19,88 & 0,007 & $29,99^{a}$ & $00^{c}$ & $16,66^{b}$ & $23,33^{\mathrm{ab}}$ & 17,49 \\
\hline \multirow[b]{2}{*}{ TV } & Au volume & 56,35 & 0,001 & $29,99^{b}$ & $93,33^{a}$ & $26,66^{b}$ & $93,33^{a}$ & 60,82 \\
\hline & sacs & 28,39 & 0,004 & $66,66^{a}$ & $6,66^{b}$ & $56,66^{a}$ & $6,66^{b}$ & 34,16 \\
\hline \multirow[b]{2}{*}{ PPS } & $5000-1000 \mathrm{f}$ & 20,39 & 0,007 & $93,33^{a}$ & $83,33^{a}$ & $43,33^{b}$ & $93,33^{a}$ & 78,33 \\
\hline & $2000-5000 \mathrm{f}$ & 20,40 & 0,007 & $6,66^{b}$ & $16,66^{b}$ & $56,66^{a}$ & $6,66^{b}$ & 21,66 \\
\hline \multirow[b]{2}{*}{ PV } & $200-500 \mathrm{f}$ & 3,56 & 0,001 & $43,33^{a}$ & $26,66^{b}$ & $43,33^{a}$ & $19,99^{b}$ & 33,32 \\
\hline & $50-200 \mathrm{f}$ & 4,21 & 0,002 & $56,66^{b}$ & $73,33^{a}$ & $56,66^{b}$ & $79,99^{a}$ & 66,66 \\
\hline \multirow{4}{*}{ GAP } & $300000 \mathrm{f}$ & 20,39 & 0,007 & $56,66^{\mathrm{ab}}$ & $46,66^{b}$ & $16,66^{c}$ & $73,33^{a}$ & 48,32 \\
\hline & $200000 \mathrm{f}$ & 14,24 & 0,013 & $26,66^{b}$ & $23,33^{b}$ & $49,99^{a}$ & $9,99^{b}$ & 27,49 \\
\hline & $150000 \mathrm{f}$ & 2,86 & 0,16 & $13,33^{a}$ & $26,66^{a}$ & $16,66^{a}$ & $13,33^{a}$ & 17,49 \\
\hline & $100000 \mathrm{f}$ & 1,22 & 0,41 & $3,33^{a}$ & $3,33^{a}$ & $9,99^{a}$ & $6,66^{a}$ & 5,82 \\
\hline
\end{tabular}

Les chiffres ayant la même lettre en exposant ne sont pas significativement différents

VPD : Vendeur de la patate Douce ; APD : Acheteurs de la patate douce ; LV : Lieu de vente ; TV : Type de vente ;

PPS : Prix par sac ; PV : Prix au volume ; GAP : Gain annuel du producteur.

Difficultés rencontrées dans la production de la patate douce: La figure 2 présente les difficultés rencontrées dans la production de la patate douce. De cette figure, on constate que les difficultés les plus marquantes sont le manque des intrants et le manque des pluies. A Moulkou et Gournaida, la difficulté la plus importante connue dans la production de la patate douce est le manque des intrants soit 76,66\% des enquêtés le déclarent. Par contre à Bariam 63,33 \% des producteurs disent que la difficulté la plus importante notée est le manque de la main d'œuvre. Tandis qu'à Guizédé, 36,66 \% des producteurs notent le manque des pluies et intrants comme difficultés rencontrées dans la production de la patate douce. 


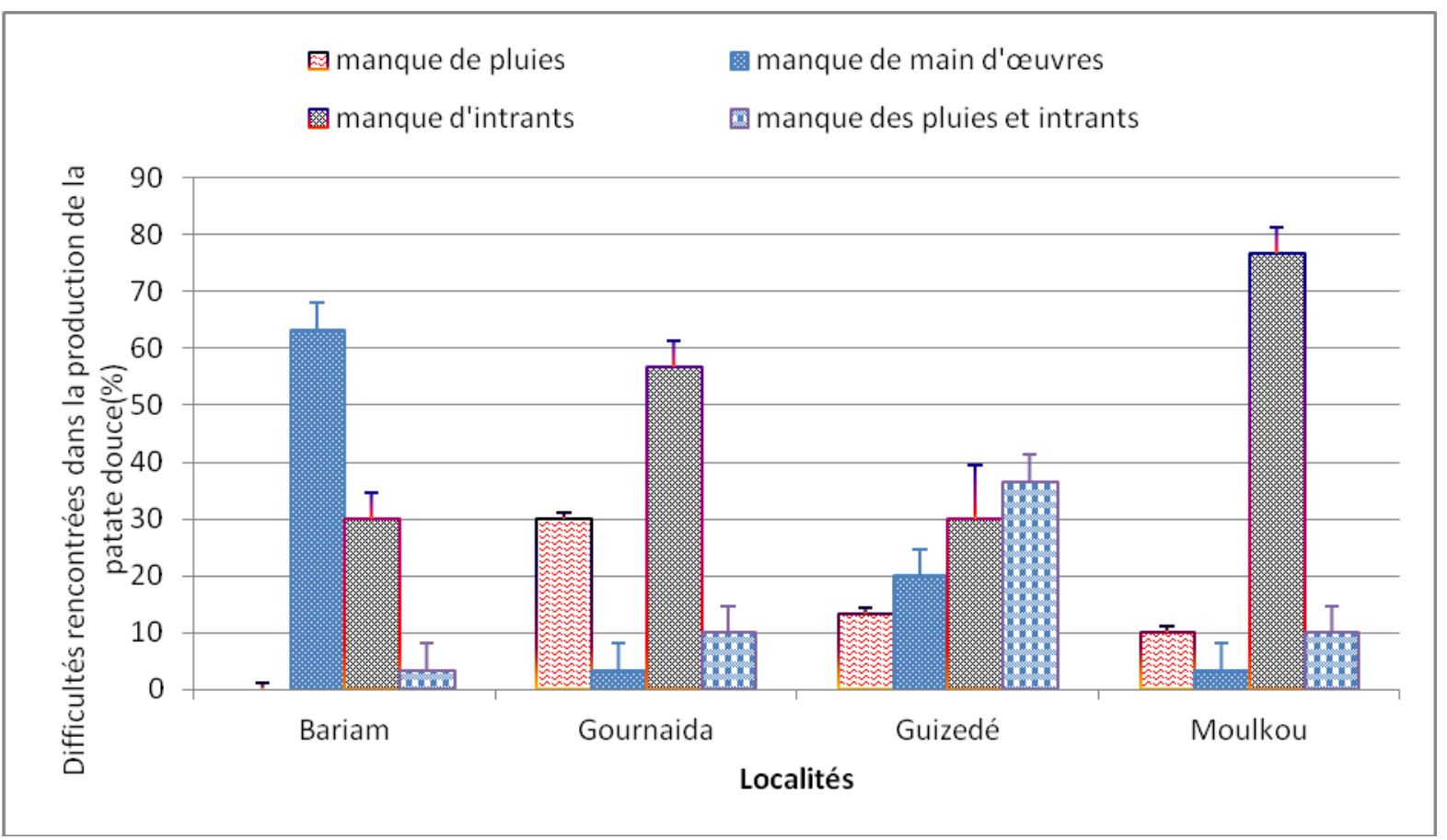

Figure 2 : difficultés rencontrées dans la production de la patate douce

\section{DISCUSSION}

La culture de la patate douce se fait au moyen des boutures. Les boutures sont produites soit par les cultivateurs eux-mêmes à partir des tubercules gardés en réserve ou des fragments de tige mis en pépinières dans les petits jardins autour des cases soit par les multiplicateurs spéciaux de la place ou d'ailleurs qui les vendent dans les sacs à l'approche de la période de plantation. Les multiplicateurs entretiennent les boutures dans les enclos pendant au moins 2 mois avant d'être récoltés entre juillet et août en vue de la plantation. II faut aussi noter que la plupart des boutures proviennent de Zébé et Herdin au Cameroun, des villages séparés de la ville de Bongor par le fleuve Logone. Les multiplicateurs de cette localité se mettent en groupement pour pouvoir mener cette activité. Cependant, les producteurs n'utilisent pas un nombre important de variétés qui sont reconnues par les feuilles (forme et couleur), les tiges (couleur et longueur) et les tubercules (couleur de la peau et la pulpe, la forme). Ils manifestent une préférence pour des variétés ayant la peau blanche aussi bien que la pulpe; ceci pour répondre aux gouts des acheteurs et consommateurs de patate douce. Mais le manque de boutures demeure un problème fondamental. Les critères de reconnaissance décrit par les producteurs vont en accord avec ceux décrit par (Doussoh et al., 2016) dans la production de la patate douce au Benin. En outre, 2 variétés sont généralement utilisées par la plupart des producteurs. Certaines variétés sont abandonnées car elles donnent plus la biomasse aérienne que les racines importantes défavorisant ainsi le rendement en tubercules. L'utilisation d'un nombre important de variétés s'avère nécessaire pour améliorer le rendement en milieu paysan. Au Tchad, la production des boutures de la patate reste informelle. Dans les pays comme le Burkina Faso, il existe des structures spécialisées dans la production de semences améliorées de patate douce. C'est le cas de l'INERA à Kamboinsé(Ouagadougou). La culture de la patate douce se fait sur la base des boutures plantées sur les buttes ou sur les billons. La plantation se fait naturellement par fragment des boutures dont la taille varie entre 3 et 4 entre-nœuds. Les producteurs de la patate douce des différentes localités utilisent des buttes au détriment des billons. Ces derniers disent que faire des billons est plus pénible que faire les buttes; même en termes de dépense, la butte revient moins chère que le billon. Les écartements entre les buttes ou billons sont environ $50 \mathrm{~cm}$. Sur les buttes les boutures sont enfouies à la main. Habituellement 2 à 6 boutures sont plantées sur chaque butte. Cette technique culturale va en accord avec celle décrite par Jana (1982). Mais ce dernier affirme que l'espacement influe peu sur les rendements à l'hectare mais les 
écartements plus larges donnent des racines plus grandes. Cependant Bourke (1982) dans ses études a montré que, lorsque la culture de la patate douce est faite sur billon, ceux-ci sont espacés de 0,90 à $1,5 \mathrm{~m}$, les tiges se développent bien mais le rendement est faible. Cela témoigne la préférence des producteurs pour les buttes. Le cycle des variétés utilisées tourne au tour de 3 mois et la plupart des producteurs font cette activité depuis plus de 10 ans et ils affirment que les femmes sont bel et bien impliquées dans le domaine. Mais pour elles, la tâche est ardue et onéreuse. Au Congo bien que la patate douce est exploitée à petite échelle, mais ce sont les femmes qui font revivre l'activité car elle joue un rôle prépondérant dans la sécurité alimentaire familiale de sorte que dans le Bushi (région de la province orientale du Sud Kivu), elle porte le nom de « cilera Bana » qui signifie gardien des enfants c'est-à-dire sa présence permet d'assurer la survie des enfants (Mutombo et al., 1999). Cependant dans la production de la patate douce, les fumures d'origines minérales et organiques sont utilisées pour améliorer le rendement. L'étude a aussi montré que la fumure d'origine minérale est beaucoup plus utilisée par les producteurs des différentes localités. Celle d'origine organique n'est pas utilisée pourtant les producteurs sont aussi des grands éleveurs mais très peu de ceux-ci utilisent les déchets des animaux pour pouvoir amender le sol. Une sensibilisation dans le domaine s'avère nécessaire pour améliorer le rendement. La quantité de fumures épandues est environ $10 \mathrm{~kg}$ à l'hectare. La plupart des producteurs de patate douce la cultivaient autrefois principalement pour l'autoconsommation mais aujourd'hui la majorité des producteurs orientent une grande part de la récolte vers le marché pour satisfaire leurs besoins et une petite partie est gardée pour la consommation familiale. La culture de la patate douce est devenue une activité génératrice de revenue surtout avec le changement climatique qui a un impact négatif sur les autres cultures vivrières. La récolte se fait trois mois après plantation selon les variétés. La fréquence de récolte est variable et ceci selon la taille du champ et selon la demande sur le marché ; plus la demande est élevée, plus la fréquence est élevée, la demande est moindre et la fréquence est échelonnée. Selon Bourke(1982) la méthode de récolte la plus répandue est aussi la récolte échelonnée car les grosses racines sont déterrées au fur et à mesure des besoins. Et, elle permet aussi à certaines racines de se développer. Mais à un moment donné, le producteur est contraint de vendre sa production même si la demande est faible faute de conditions de stockage. Les tubercules de patate douce se conservent difficilement, certains producteurs utilisent des fosses dans lesquelles est mis le son de penicillaire (Pennisetum typhoides). Les producteurs utilisant cette technique affirment que les tubercules peuvent y rester 3 à 4 mois. Cependant d'autres conservent les tubercules en champ sur les buttes ou billons en utilisant de produits phytosanitaires pour empêcher l'attaque de certains ravageurs. Au Benin, la conservation se fait sur les billons, dans un endroit humide et dans une moindre mesure dans un trou (Doussoh et al., 2016). La même technique est utilisée en Papouasie Nouvelle-Guinnée mais le trou est rempli de sable (Bourke, 1982). Par contre au Niger, les tubercules sont conservés à l'ombre des arbres, dans les greniers (Harouna Issa et al., 2015). Par ailleurs au Zimbabwé, les producteurs utilisent de la cendre pour conserver les tubercules car elle a un effet répulsif sur les ennemies de la patate et absorbe aussi l'humidité. La conservation de la patate douce constitue un problème fondamental. Les conditions idéales de stockage des patates douces consistent à maintenir une température de $30^{\circ} \mathrm{C}$, une humidité relative de 85 à $90 \%$ et aération importante (Wilson et Brams, 1982). Bien que plusieurs techniques de stockages sont utilisées mais les résultats restent toujours non satisfaisants car les tubercules de patate douce ont une peau assez fragile qui les rend relativement vulnérables (Harouna Issa et al., 2015). Une partie des tubercules conservés ou non est consommée sous forme frite ou bouillie. Mais c'est sous forme bouillie que la patate douce est consommée dans le milieu paysan. Cette préférence des producteurs est aussi liée à leur revenu disent-ils car frire la patate demande une quantité importante d'huile alors que le paysan vend ses arachides crues. Ils achètent la patate frite chez les commerçantes détaillantes qui font cette activité dans les petits marchés de la place. Tandis qu'à Taiwan les patates douce fraiches sont mélangées avec du riz blanc; elles sont consommées occasionnellement sous forme frite ou grillées au charbon du bois (Chen, 1982). Certains d'entre eux apprécient la patate frite surtout ceux qui sont à proximité de la ville. Outre la patate douce, les producteurs s'intéressent aussi aux cultures vivrières telles que l'arachide, le sésame, le mil .Et cela ne leur permet pas d'utiliser une grande superficie pour la culture de la patate douce. Ainsi dans le milieu paysan le rendement varie entre 2,6 et 13 tonnes à l'hectare. Ces valeurs sont proches de celles obtenues par Djinet et al . (2016) sur dix(10) variétés 
de patate douce cultivées à Bongor dans les conditions expérimentales dont les valeurs varient entre 0,94 et 45,5 t/ha. Tandis que Jana (1982) a obtenu 2,5- 15 $T /$ ha de racines fraiches. Les tubercules récoltés sont vendus, la vente de la patate se fait par le producteur lui-même soit sa femme soit les deux. Beaucoup des producteurs préfèrent vendre leurs produits de récolte eux-mêmes sous prétexte que la femme va utiliser l'argent à d'autres fins. Elle se fait généralement sur les marchés de la place ou sur les marchés des villages voisins. La demande de la patate douce généralement plus forte sur les marchés urbains que ruraux, les producteurs écoulent leur récolte vers la ville. La plus grande partie du produit est écoulée auprès des consommateurs et commerçants revendeurs venant des grandes agglomérations. Ceux-ci savent faire la différence et en apprécient la valeur. Une enquête menée à Taiwan a aussi montré que près de $2 / 3$ des patates récoltées par les agriculteurs suivaient les circuits de commercialisation, et que le tiers restant était utilisé par les agriculteurs eux-mêmes (Chen, 1982). La patate est vendue soit par sac, soit au volume. La vente au volume est plus appréciée par les producteurs de Gournaida et Moulkou car elle est bénéfique, génère plus d'argent et permet d'écouler rapidement le produit car le prix est accessible à tout le monde. II faut dire que ce type de vente est rendu facile du fait qu'il existe un grand marché hebdomadaire dans chacune des localités. Tandis que les producteurs de Bariam préfèrent vendre leurs produits par sac ; cette tendance s'explique par le fait que la localité est à proximité de la ville de Bongor et l'accessibilité est plus facile. Cependant les prix des produits sont fixés en fonction de la demande, des saisons de l'année et de la qualité des tubercules. Les caractéristiques les plus importantes sollicitées par les consommateurs et les acheteurs ou commerçants sont la forme et la couleur de l'enveloppe ou de la peau. C'est ainsi que les

\section{CONCLUSION}

La production de la patate douce se fait au moyen des boutures. Les boutures sont issues des tubercules ou des tiges mises en pépinière par les producteurs euxmêmes ou les multiplicateurs spéciaux chez lesquels les producteurs achètent en vue de la plantation pendant le mois de juillet. Dans la pratique culturale les producteurs utilisent plus des buttes que les billons avec l'écartement de $50 \mathrm{~cm}$ entre les unes et les autres et la plantation à plat est rare. Dans la plupart des cas, les variétés dont le cycle est de 3 mois sont cultivées. Et dans les localités faisant l'objet d'étude, les tubercules blancs sont plus vendus. Les producteurs respectent donc l'exigence des acheteurs et consommateurs. En outre, il semble que les variétés ayant la peau rouge se conservent difficilement et n'ont pas des caractéristiques requises du point de vue organoleptiques. Par contre selon Hayma(2004), les variétés rouges semblent mieux se conserver que les blanches. Cette différence peut être liée aux facteurs du milieu dans lequel la patate a été expérimentée. Le gain annuel des producteurs va de 100.000 à 300.000 f. Ce gain permet aux producteurs de subvenir aux besoins de la famille. II faut noter aussi que les localités faisant l'objet d'études étaient autrefois des zones de production de coton par excellence mais depuis plus de deux décennies cette culture est abandonnée au détriment des autres cultures. En marge des marchés, les secteurs de la commercialisation de la patate douce au Tchad demeurent très primitifs, composés en majorité par de très petits producteur-revendeur qui écoulent leurs produits à dos d'âne ou à vélo ou à l'aide de tricycle sur des petits marchés souvent très éphémères. Dans la production de la patate douce les producteurs rencontrent d'énormes difficultés. Parmi les difficultés, on peut citer le manque de pluies, le manque d'intrants, le manque de mains d'œuvres, pluies et intrants. Mais le problème fondamental causant la baisse de rendement est lié au manque de pluie et intrants selon les producteurs. Le prix d'intrant n'est pas à la bourse de tous les producteurs, il est difficile de s'en procurer, et la période d'épandage se situe entre Juillet et Août considérés par les producteurs comme les mois de soudure. Pendant cette période les moyens deviennent rares beaucoup des producteurs n'arrivent pas à acheter des intrants. Le manque des pluies signalé comme difficulté majeure dans la production de la patate douce va en accord avec ce que Doussouh et al. (2014) ont noté aussi dans la production de la patate douce au Benin.

producteurs pratiquent cette activité depuis plus de 10 ans. Cependant dans la production de la patate douce, les fumures d'origine minérale sont beaucoup plus utilisées pour augmenter les rendements. En outre, la fréquence de récolte de la patate est fonction de la taille du champ et de la demande sur le marché. Le rendement varie entre 2,6 et 13 tha dans le milieu paysan traditionnel. Les tubercules récoltés sont directement vendus faute de moyen de conservation. Certains producteurs utilisent les fosses dans lesquelles est mis le son de Pennisetum thyphoides 
pour conserver les tubercules. Dans le milieu paysan, la patate est consommée sous forme bouillie. Mais la plus grande partie de la récolte est écoulée auprès des consommateurs et commerçants venant des grandes villes. Par ailleurs, la vente se fait soit par sac, soit au volume et le prix par sac varie entre 5000 et $10000 \mathrm{~F}$ alors que celui au volume varie entre 200 et $500 \mathrm{~F}$. Mais le gain annuel des paysans va de 100.000 à $300.000 \mathrm{~F}$ pendant la campagne. Pour répondre aux exigences des acheteurs et consommateurs les producteurs préfèrent les tubercules blancs. Cependant

\section{REMERCIEMENTS}

Nos sincères remerciements aux élève-professeurs de la troisième année des Sciences de la Vie et de la Terre de l'Ecole Normale Supérieure de Bongor pour

\section{REFERENCES BIBLIOGRAPHIQUES}

Bourke RM, 1982. La patate douce en Papouasie Nouvelle-Guinnée. In Patate douce. CTA/ ACCT. Shanhua ; 45-47.

Bovell-Benjamin AC, 2017. Sweet potato: a review of its past, present and future role in human nutrition. Adv. Food. Nutr. $52: 1-59$.

Chen HY, 1982. La commercialisation des patates à Taiwan. In Patate douce. CTA ACCT. Shanhua ; 45-47.

Djinet IA, Nana R, Tamini Z, Badiel B, 2015. Etude compare des paramètres agromorphologiques de dix(10) variétés de patate douce (Ipomoea batatas (L.) Lam. Cultivées au champ dans deux conditions climatiques au Tchad et Burkina Faso. Int. J. Biol. Chem. Sci., 9(3) : 1243-1251.

Doussoh MA, Dagou SJ, Houedjissin SS, Assogba KA, Ahanhanzo C, 2016. Analyse des connaissances endogènes et des déterminants de la production de la patate douce [Ipomoea batatas (L.)], une culture à haute valeur socioculturelle et économique au Benin. Int. J. Biol. Chem. Sci., 10(6) : 25962616.

Dossou-Aminon I, Loko LY, Adjatin A, Dansi A, Elangovan $M$, Chaudhary $P$, Vodouhe $R$, Sanni A, 2014. Diversity, genetic erosion and dans la production de la patate douce, la difficulté la plus importante est le manque des pluies et intrants. Ainsi dans les perspectives, une autre enquête va être menée dans les marchés de la ville de Bongor, pour comparer la plus-value des commerçants revendeurs et celle des producteurs de la patate douce. En outre, une autre enquête peut être aussi menée dans une autre zone agro-écologique du Tchad pour mieux connaitre les pratiques agricoles adoptées par les producteurs dans leur milieu afin de pouvoir chercher à améliorer le rendement de la patate douce.

les enquêtes menées dans les différents villages. Nous tenons également à remercier l'INERA pour son soutien matériel.

farmers preference of sorgum varieties [Sorghum bicolor (L.) Moench] in NorthEastern Benin. Int. J. Curr. Microbiol. Appl. Sci., 3(10): 531-552.

Harouna Issa A, Doumma A, Toufigue Bello M, 2015. Inventaire des varieties, des methods locales de stockage et de protection contre les ravageurs de la patate douce (Ipomoea batatas L.) dans la bande ouest du Niger. Int. J. Biol. Chem. Sci., 9(4) : 1962-1971.

Jana RK, 1982. La situation de la culture de la patate douce en Afrique orientale et son avenir. In Patate douce. CTA/ACCT. Shanhua; 63-76.

Kaola M, Hema A, Somé K, Palé E, Sérémé A, Belem J, Nacro M, 2013. Evolution of eight orange flesh sweet potato (OFSP) varieties for their total antioxidant, total carotenoid and polyphenolic content. J. Nat. Sci. Res., 3(4): 67-72.

Mutombo T, Phemba P, Feruzi M, Lutaladio NB, Ewel $\mathrm{P}, 1$ 1999. Pomme de terre et patate douce au Bushi. CERPR/ISDR, Bukavu.

Vernier P, Varin D, 1994. La culture de la patate douce. Agri. Dev, 3(1): 54-63.

Wilson LG, Abrams JR, 1982. La mécanisation de la production de la patate douce. In Patate douce. CTA/ACCT. Shanhua ; 63-76. 